\title{
Los medios de comunicación y la pedagogía sobre el significado de la violencia machista
}

\author{
Komunikabideak eta pedagogia, indarkeria \\ matxistaren esanahiaren inguruan
}

\author{
Mass media and pedagogy \\ on meaning of gender violence
}

\section{Manuel Peris Vidal ${ }^{1}$}

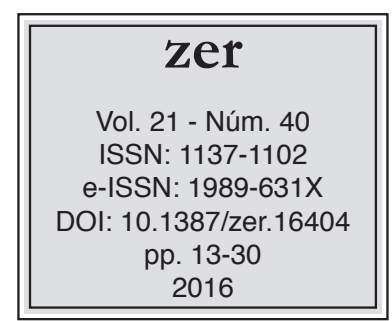

Recibido el 16 de agosto de 2015, aceptado el 11 de enero de 2016.

\section{Resumen}

Los tres principales diarios españoles no incluyen en sus noticias prácticamente ningún comentario explicativo que vincule la violencia machista con la discriminación que sufren las mujeres. Este aspecto resulta relevante porque se considera que no es suficiente con el seguimiento de las principales recomendaciones sobre el tratamiento mediático de la violencia, y que las declaraciones procedentes de fuentes expertas o las reflexiones de los periodistas deben poseer un carácter pedagógico. Con este fin, se ha construido un indicador para medir la labor de difusión del verdadero significado de la violencia de género.

Palabras clave: Violencia machista, pedagogía, representación mediática, fuentes expertas, desigualdad de género.

\section{Laburpena}

Espainiako hiru egunkari nagusiek ez dute albisteetan ia ezer esaten indarkeria matxistaren eta emakumeek jasaten duten diskriminazioaren arteko lotura azaltzeko. Alderdi hori azpimarragarria da; izan ere, gure iritziz, ez da nahikoa komunikabideetan indarkeria tratatzeko gomendio nagusiei jarraitzea. Horrez gain, adituengandik jasotako adierazpenek edo kazetarien hausnarketek izaera pedagogikoa izan behar dute. Helburu horrekin, adierazle bat egin da genero-indarkeriaren benetako esanahia ezagutarazteko lana neurtzeko.

Gako-hitzak: Indarkeria matxista, pedagogia, hedabideetako irudikapena, iturri adituak, genero-desberdintasuna.

\footnotetext{
1 Universidad Pablo de Olavide de Sevilla, mperisvidal@hotmail.com
} 


\begin{abstract}
The three main Spanish newspapers contain practically no explanatory commentary that links gender violence to discrimination suffered by women. This aspect is relevant because we believe that it is not enough to follow up on the recommendations about the media portrayal of gender violence, and we believe that the statements coming from expert sources, or the reflections made from journalists should be pedagogical. To that end, we have developed an indicator to measure the dissemination work of the true meaning of gender violence.
\end{abstract}

Keywords: Gender violence, pedagogy, media portrayal, expert sources, gender inequality. 


\section{Introducción}

Desde nuestra perspectiva, los medios de comunicación no se deben limitar a transmitir con objetividad los hechos más relevantes de las agresiones machistas sobre las que informan, sino que sería conveniente que llevaran a cabo una labor pedagógica para difundir el verdadero significado de la violencia de género, si tenemos en cuenta que todavía existen importantes carencias en la comprensión de este problema por parte de la ciudadanía. Para ello, sería conveniente que las noticias de la prensa escrita relacionaran este tipo de violencia con el orden patriarcal, el cual, según expone Kate Millet en Sexual Politics (1970), incluye dos componentes básicos: una estructura social, que es el sistema de organización social que crea y mantiene una situación en la que los hombres tienen más poder y privilegios que las mujeres, y una ideología o conjunto de creencias que legitima y mantiene dicha situación (Bosch y Ferrer, 2013: 57). Esta ideología sería la que se emplea para justificar la violencia contra las mujeres que violan los ideales de la familia patriarcal y que, por lo tanto, pondrían en peligro la posición de dominio de los maltratadores.

Así pues, el concepto de violencia machista que se debería difundir a través de los mass media es el que está presente, entre otras, en la definición expuesta en el párrafo 118 de la Plataforma de Acción de Beijing, aprobada durante la IV Conferencia Mundial sobre las Mujeres de 1995, organizada por la ONU: "La violencia contra las mujeres es una manifestación de las relaciones de poder históricamente desiguales entre mujeres y hombres, que han conducido a la dominación de la mujer por el hombre, la discriminación contra la mujer y a la interposición de obstáculos contra su pleno desarrollo" (Organización de Naciones Unidas, 1995: 52).

Aunque este concepto debería ser divulgado también desde otros ámbitos, resulta especialmente relevante el papel de los medios de comunicación en este asunto, si atendemos a los datos del informe del Special Eurobarometer 344/ Wave 73.2, titulado Domestic Violence against Women Report, encargado por la Comisión Europea y elaborado en el año 2010. Según este informe, la mayoría de la población de España y de Europa ha oído hablar de la violencia machista a través de la televisión, de los periódicos, las revistas o la radio. En la encuesta, se planteó la siguiente pregunta de respuestas múltiples: "¿Ha oído alguna vez hablar de la violencia doméstica contra las mujeres? ¿Dónde oyó hablar sobre ello?” (European Commission, 2010: 14). Los resultados muestran que los porcentajes de personas europeas que han oído hablar de la violencia machista por medio de periódicos y revistas son muy elevados, con un 59\% de media en la Unión Europea y un 57\% para España. Si observamos la proporción de población que ha oído hablar de este tema a través de otros medios de comunicación, como la televisión, las cifras son todavía mayores, con un $92 \%$ de media en el caso de Europa y un 97\% para el Estado Español (European Commission, 2010: 14).

\section{La importancia de los géneros informativos en la lucha contra el machismo}

La noticia es el género periodístico utilizado con mayor frecuencia para tratar la violencia de género en la prensa escrita, según se desprende de diversas investigaciones, como la de Ariznabarreta et al. (2006: 181), o la de Menéndez (2010: 70). 
Además, la violencia de género "irrumpe mayoritariamente en las páginas de los diarios a través de noticias sobre agresiones cometidas por los maltratadores y sobre su detención" (Sanz, 2006: 98). Los lectores y las lectoras están acostumbrados, desde el comienzo de la aparición de este asunto en la prensa, a fijar su atención sobre los casos concretos que se suceden un mes tras otro. Como consecuencia, defendemos la necesidad de aprovechar la enorme audiencia potencial de las noticias para incluir en ellas aportaciones de fuentes expertas en la violencia de género o comentarios de los propios autores y autoras de las informaciones, que expliquen el verdadero origen de esta clase de violencia, o que expongan algunas medidas necesarias para su prevención. Todo ello con el fin de concienciar a la ciudadanía sobre la dimensión de este problema y sobre cuáles son sus causas.

La información como género periodístico, de acuerdo con las características que le asigna Martínez Albertos (1992: 271), es la misma noticia (o hecho que se pretende comunicar) en sus elementos básicos, acompañada de sus circunstancias explicativas, está escrita con un estilo literario sobrio y escueto, rigurosamente objetivo, y normalmente no tiene continuidad. Según esta definición, las informaciones carecerían de comentarios complementarios sobre los hechos de actualidad, puesto que el estilo rigurosamente objetivo impide, en teoría, que el autor o autora de la misma analice lo sucedido. Desde esta perspectiva, en el caso de las noticias sobre violencia machista, los y las periodistas se deberían limitar a recopilar la información procedente de las diversas fuentes consultadas y a transmitirla a los lectores y lectoras por medio de un estilo sobrio. Sin embargo, en este trabajo se comparte el punto de vista de quienes defienden que "el periodismo cumple un rol de interpretador de la realidad, no de espejo de ella" (Fernández Parratt, 2008: 78). Así pues, la interpretación estaría presente en todos los géneros periodísticos, incluidos los clasificados como informativos. Fernández Parratt, además, defiende que la clásica división entre géneros informativos y de opinión, a la que posteriormente se unieron los géneros interpretativos, se ve superada por la evolución de la profesión periodística, encaminada a la proliferación de géneros mixtos. A ello cabe añadir que, en la actualidad, numerosos investigadores en ciencias de la comunicación niegan que se pueda hablar de una frontera nítida entre textos periodísticos informativos y de opinión, y argumentan que cualquier texto periodístico lleva oculta una dosis de opinión (Fernández Parratt, 2003: 28). Consiguientemente, en el caso de las noticias sobre violencia machista, entendemos que sería recomendable la incorporación de algunos de los elementos complementarios mencionados, como determinados textos interpretativos o textos de opinión que trataran de explicar las causas de esta clase de violencia y situarla en el contexto de la dominación masculina. De lo contrario, los lectores y lectoras únicamente reciben información sobre el resultado de las agresiones, y no sobre su origen social, y los asesinatos pueden ser interpretados como sucesos inconexos.

\section{Estado de la cuestión, objetivos y metodología}

En este trabajo se parte de la hipótesis de que los mass media del Estado español nunca han manifestado un verdadero interés en desarrollar una labor pedagógica de mejora del conocimiento sobre el origen y las principales características de la violencia machista, que entendemos necesaria. 
Consideramos que se debe ir más allá del análisis de los errores en la representación mediática de la violencia machista, y que se debería valorar especialmente la incorporación de contenidos pedagógicos en las noticias sobre este problema. Una de las razones de esto último es el convencimiento de que en la actualidad todavía existe una parte importante de nuestra sociedad que no interpreta la violencia machista como un problema social generado por el sistema de dominación patriarcal. Así lo demuestran los resultados de algunos estudios sobre este tema, como el desarrollado por el equipo investigador de la Universidad de les Illes Balears entre los años 2007 y 2011. En él se llegó a la conclusión de que la comprensión de lo que es, y significa, la violencia de género no ha tenido aún la suficiente trascendencia como para haber calado y alcanzado al conjunto de la población (Bosch et al., 2012: 53). De hecho, se concluyó que ni siquiera existe un rechazo universal a la violencia machista en nuestra sociedad.

Asimismo, consideramos que las diversas investigaciones realizadas en el presente siglo sobre el tratamiento mediático de esta clase de violencia han prestado una escasa atención a este asunto. En este sentido, cabe destacar que sólo un número limitado de trabajos han hecho referencia expresa a la función pedagógica del periodismo en la comprensión de las causas de la violencia. Además, cuando lo han hecho, el tema no ha sido tratado en profundidad. Muchas de estas investigaciones se han apoyado en la teoría del framing con el fin de identificar los encuadres de la cobertura mediática de la violencia machista y, de este modo, conocer qué rasgos de este problema se destacan y cuáles se silencian. Un ejemplo de ello es el trabajo de Marín, Armentia y Caminos sobre la prensa vasca (2011: 454, 462), que presta una atención especial a los aspectos formales de la noticia y a los diferentes encuadres temáticos, pero sólo hace una referencia residual a la pedagogía sobre la violencia cuando se alude a la conveniencia de incluir fuentes expertas. Otro aspecto relacionado con la mejora del conocimiento de la violencia machista, el de la contextualización de las agresiones, también es tratado en este trabajo, aunque para resaltar que los resultados del examen son decepcionantes. El motivo radica en que, aunque entre el $48 \%$ y el $53 \%$ de las informaciones estudiadas recogen algún tipo de contenido complementario, en muchos de estos casos la contextualización se limita a aportar datos estadísticos o cronologías.

En otro de estos trabajos, Paula Carballido analiza exhaustivamente diversos encuadres noticiosos, aunque dedica una atención limitada al encuadre que está más estrechamente relacionado con la labor didáctica que pueden desarrollar los mass media sobre el origen de la violencia machista: el cultural. De hecho, forman parte de este encuadre aquellas referencias que hacen hincapié en los aspectos culturales, actitudinales y simbólicos de esta clase de violencia, y que ofrecen una definición de este problema como una expresión social de la desigualdad social entre hombres y mujeres, llevada al extremo (2010: 303).

El encuadre cultural también se menciona en la tesis doctoral de María Isabel Escribano, aunque su presencia también es residual, y se llega a afirmar que "bajo la denominación del 'encuadre cultural' los resultados de la investigación no han ofrecido datos” (2014: 138). Aunque la autora sí que otorga una gran importancia a este encuadre, ya que podría ayudar a cambiar la mentalidad de los lectores y lectoras desde los cimientos de nuestra cultura (2014: 168), apenas le dedica espacio en su investigación. 
Cabe destacar que los aspectos que podríamos calificar como elementos inadecuados presentes en la representación mediática de la violencia machista, son el núcleo de la mayoría de los trabajos realizados sobre esta materia, como sucede en el caso de la tesis doctoral de Elena Roselló. En ella se estudian algunos de estos factores, como los estereotipos asociados al perfil de las víctimas y de los agresores, o las referencias a las adicciones y a los problemas mentales del maltratador (2009: 155). En esta tesis sí que se muestra cierto interés por el papel pedagógico de los medios cuando se afirma que los medios de comunicación no están contribuyendo a hacer un análisis en profundidad sobre las verdaderas causas estructurales y culturales de la violencia de género (2009: 77). Sin embargo, no se detallan los posibles contenidos pedagógicos existentes en las noticias analizadas.

Otro de los trabajos que se centra en el análisis de las incorrecciones en la representación de la violencia machista es el de María Isabel Menéndez sobre la prensa balear. A pesar de la focalización sobre estos aspectos, la autora también se refiere en su estudio a la pedagogía sobre la violencia de género cuando afirma que sería deseable que, junto a la noticia del asesinato de una mujer, el público pudiera conocer algo más sobre la violencia machista como fenómeno estructural (2010: 98). Sin embargo, no se profundiza en la puesta en práctica de esta recomendación, y tampoco existe una medición específica de este tema.

Desde nuestro punto de vista, los estudios sobre la representación mediática de la violencia machista deberían ir más allá del análisis de los elementos inapropiados presentes en las noticias, y examinar también la existencia de contenidos con valor pedagógico, con el fin de contribuir a mejorar el nivel de formación periodística en materia de igualdad de género. El hecho de que todavía exista un gran número de informaciones que representan incorrectamente el problema demuestra, a nuestro juicio, que el desconocimiento del significado de la violencia machista no sólo afecta a la audiencia de los mass media, sino también a los y las profesionales que construyen las noticias. Para evitar que se produzca un efecto de retroalimentación en este aspecto, consideramos necesario que la información rigurosa sobre el significado de este problema social esté presente en el mayor número posible de textos periodísticos.

Teniendo en cuenta la importancia que otorgamos a los géneros informativos de la prensa escrita de cara a la concienciación social sobre la violencia machista, en este trabajo nos hemos propuesto alcanzar los objetivos siguientes:

- Elaborar un indicador que permita medir la contribución de la prensa escrita a la difusión, entre la ciudadanía, de un concepto preciso de lo que significa la violencia de género, es decir, conocer en qué medida las informaciones de los diarios están siendo de utilidad pedagógica para que los lectores conozcan el verdadero origen de la violencia machista y sus principales rasgos.

- Conocer el porcentaje de noticias de los tres principales diarios españoles de información general que, durante 2015, han incorporado contenidos explícitos que contribuyen a mejorar el conocimiento de la ciudadanía sobre la violencia machista. 
- Comprobar si la presencia de mensajes de carácter pedagógico en las noticias es una consecuencia de la actitud comprometida de la persona que elabora la información o, por el contrario, es fruto de la casualidad.

- Invitar a reflexionar sobre la necesidad de aumentar el nivel de exigencia en el tratamiento de la violencia machista en los medios de comunicación, que vaya más allá de la mera representación rigurosa de este problema, y que incluya una actitud comprometida por parte de los autores y las autoras de las informaciones.

Con vistas a la consecución de este último objetivo, se han escogido los diarios El País, El Mundo y ABC, dado que hemos entendido que son los tres periódicos más representativos en el ámbito del conjunto del Estado español, por tratarse de los diarios de información general con mayor promedio de tirada entre enero y diciembre de 2014, además de estar situados, respectivamente, en el primer, segundo y cuarto lugar en cuanto al promedio de difusión de dicho tipo de prensa durante ese mismo período ${ }^{2}$.

En este estudio se han analizado las primeras noticias de cada uno de los casos de violencia machista con resultado de muerte en el ámbito de las relaciones de pareja o similares, que hayan sido publicadas en estos tres diarios durante 2015. Para ello se han utilizado los buscadores de las ediciones digitales de El País (http://elpais. com/buscador/), El Mundo (http://www.elmundo.es) y $A B C$ (http://www.abc.es). La muestra está constituida por 109 informaciones (36 de ABC, 37 de El Mundo y 36 de El País).

Hemos considerado que el corpus seleccionado es significativo, en parte, por ser muy reciente, y porque entendemos que la elección de la primera noticia sobre cada uno de los asesinatos machistas del período analizado representa adecuadamente al conjunto de las informaciones de estos diarios, ya que la prensa presenta un alto grado de redundancia. Además, el hecho de que el análisis abarque un año entero puede evitar que la muestra seleccionada se vea afectada por determinadas situaciones coyunturales que pudieran generar modificaciones pasajeras en el enfoque periodístico, como podría ser una concentración anormal de asesinatos machistas en un momento concreto.

El motivo de la limitación del examen a aquellos casos de violencia con víctimas mortales y que, además, se hayan producido en el contexto de las relaciones de pareja o similares, radica en que los medios de comunicación españoles suelen limitar sus informaciones sobre la violencia de género a dichas situaciones, de manera que son silenciados habitualmente, tanto los miles de agresiones machistas sin resultado de muerte producidas en nuestro país cada año, como la violencia física, sexual o psicológica ocasionada por hombres que no son la pareja o expareja sentimental de las víctimas. En las informaciones periodísticas sobre la violencia de género se promueve la utilización de un concepto limitado de este problema entre la ciudadanía, que suele excluir, además, asuntos como la intimidación sexual en el trabajo, la trata de mujeres o la mutilación sexual.

\footnotetext{
${ }^{2}$ Según datos de la Oficina de Justificación de la Difusión (OJD): http://www.introl.es/medios-con-
} trolados/, 21/07/2015. 
En la parte prescriptiva del presente análisis se ha llevado a cabo una reflexión crítica sobre la función de los mass media en la mejora del conocimiento sobre la violencia machista, con el fin de establecer cuáles son los mensajes sobre el origen de este problema que sería recomendable incorporar en las informaciones sobre esta clase de violencia.

En cuanto a la parte descriptiva de este estudio, se ha adoptado la perspectiva metodológica del análisis crítico del discurso, definido por Norman Fairclough (2003: 181) como "el análisis de las relaciones dialécticas entre la semiosis (incluido el lenguaje) y otros elementos de las prácticas sociales". Esta perspectiva nos ha parecido adecuada para el tema del presente estudio porque consideramos que los discursos sólo pueden entenderse por referencia a su contexto, y compartimos la posición de Ron Scollon (2003: 206) cuando afirma que los problemas sociales se constituyen en gran medida por medio del discurso. En nuestro caso, se han analizado lo que Teun A. Van Dijk (2003: 152) denomina "temas" o "macroestructuras semánticas", que son el significado global que los usuarios de una lengua establecen mediante la producción y la comprensión de discursos, y cuya relevancia social radica en que definen el elemento hacia el que se orientan los y las hablantes, las organizaciones y los grupos, y a través de ellos los y las hablantes y escritores/as pueden influir en la formación de los "modelos mentales" del acontecimiento que aborda el discurso. Estas macroestructuras semánticas se han examinado en el presente trabajo, por un lado, al comprobar si la violencia machista es representada en la prensa como un suceso o como un problema social. Por otro lado, el análisis central de los temas se ha dirigido a la cuantificación de los contenidos de carácter pedagógico. Estas estructuras del discurso se han puesto en relación con lo que Van Dijk (2003: 160) denomina "estructuras de los contextos locales y globales". En nuestro caso, el contexto global - que se define por las estructuras sociales, políticas, culturales e históricas en las que tienen lugar los acontecimientos comunicativos (Van Dijk, 2003: 161) - sería el de un orden patriarcal que legitima y sostiene el poder de los hombres sobre las mujeres, porque argumenta la superioridad de los unos y la inferioridad de las otras, "dando así carta de naturaleza a la subordinación de ellas a ellos en la sociedad y en la familia" (Bosch et al., 2013: 272). Esta ideología es un elemento clave en la explicación de la violencia machista como estrategia de control. Sin embargo, en el contexto de los medios de comunicación españoles no siempre se construyen los significados a partir de esta perspectiva, sino que los casos de violencia de género son tratados como hechos aislados sin conexión con la dominación masculina, y debidos a causas diversas. Por todo ello, hemos considerado oportuno estudiar los vínculos entre el texto y el contexto a partir del análisis crítico del discurso.

\section{Una propuesta de medición de la pedagogía sobre la violencia machista}

En el presente trabajo se propone un indicador que permita medir la labor pedagógica que se lleva a cabo desde la prensa escrita para mejorar el conocimiento ciudadano sobre la violencia de género. Concretamente, se pretende medir el siguiente concepto: Contribución de la prensa escrita a la divulgación de la noción de la violencia machista como una manifestación de la discriminación que sufren las mujeres. 
En primer lugar, cabe destacar que en los códigos de autorregulación y manuales de recomendaciones sobre el tratamiento de la violencia de género en los medios de comunicación que se han elaborado desde finales del siglo xx en España, sólo se ha hecho referencia de manera esporádica a la conveniencia de incluir, en las noticias sobre este asunto, datos objetivos o reflexiones de fuentes expertas que mejoren el nivel de conocimiento de la ciudadanía sobre la violencia machista. A continuación se exponen algunos de los escasos ejemplos de dichas sugerencias:

- Hay que ofrecer siempre, en la noticia, los testimonios de personas expertas en Violencia contra las Mujeres, como, por ejemplo, diversas asociaciones de mujeres, así como la opinión de policías, psicólogos/as y jueces y juezas, que deben hablar como profesionales representantes del estamento al que pertenecen y condenar los hechos (Bandrés, 2011: 133134 [pauta número 5]).

- Es conveniente buscar fuentes cualificadas y especializadas para las noticias relativas a la violencia de género, dar voz a profesionales de servicios y entidades especializadas. Algunas de estas fuentes son servicios de atención a mujeres que sufren violencia, asociaciones de mujeres agredidas, centros de asesoramiento jurídico y/o psicológico específico para mujeres, hospitales, centros de salud y centros de recuperación de maltratadores (Col-legi Oficial de Periodistes de Catalunya et al., 2004: 11 [recomendación número 4]).

- Es conveniente ampliar y contextualizar la información sobre la violencia de género con aportaciones que ayuden a visibilizar el problema en su conjunto, como datos contrastados y acumulativos que permitan reflejar la dimensión del fenómeno (Col·legi de Periodistes de Catalunya et al., 2004: 14 [recomendación número 10]).

Empero, la puesta en práctica de estas recomendaciones ha supuesto, por un lado, que numerosos diarios se hayan limitado a incluir declaraciones intrascendentes de fuentes expertas que no ayudan a divulgar el verdadero significado de la violencia de género, o meras declaraciones de condena de los asesinatos, procedentes de fuentes expertas o de fuentes institucionales. Y, por el otro, que muchos de estos medios hayan aportado las cifras del número de mujeres asesinadas durante el año en curso o durante un período temporal concreto, pero sin añadir comentarios explicativos sobre aquéllas, de manera que los datos sobre el número de víctimas se convierten en un elemento rutinario que va unido a las informaciones de manera casi automática y que se convierte en uno más de los elementos fijos de la estructura de la noticia.

Como consecuencia de todo ello, en este estudio sólo se han valorado positivamente las declaraciones procedentes de fuentes expertas si incluyen explicaciones sobre el verdadero significado de la violencia de género. Asimismo, se consideran provechosas las aportaciones de cualquier otra fuente utilizada en la noticia, o las 
del autor o autora de la noticia, siempre que permitan comprender mejor el significado de la violencia machista, o cuando dichas reflexiones complementan los datos sobre mujeres asesinadas con el fin de mostrar la dimensión del problema o su carácter estructural. Así pues, se considera que las informaciones que contribuyen a difundir la noción de la violencia machista como una manifestación de la desigualdad de género, son aquellas que incluyen algunos de los siguientes elementos:

- Comentarios complementarios del autor o autora de la información, que relacionen directamente la violencia machista con la discriminación que sufren las mujeres. Tales comentarios pueden hacer referencia a la desigualdad en la distribución de roles sociales; también pueden aludir al origen de la violencia en la estructura social fundada sobre las bases del domino patriarcal; o vincularse al hecho de que la violencia machista es un recurso empleado por los hombres para garantizar su relación de dominio y, así, mantener a las mujeres bajo su control absoluto y garantizar la continuidad de un orden tradicional de valores discriminatorios, los cuales perpetúan la subordinación de las mujeres a la autoridad masculina. También se valorarán positivamente las alusiones a las medidas preventivas en materia de educación o a la regulación y autorregulación de los contenidos y la publicidad en los medios de comunicación en el ámbito de esta clase de violencia. Asimismo, se incluye en este indicador la presencia de cifras de agresiones, asesinatos y denuncias por violencia de género, siempre que vayan acompañadas de explicaciones sobre las particularidades de este tipo de violencia, de manera que ofrezcan al lector una idea aproximada de las proporciones epidémicas del problema.

- Aportaciones procedentes de todo tipo de fuentes que relacionen directamente la violencia machista con la desigualdad de género, o incorporación de datos científicos sobre esta materia. Se ha valorado la presencia de informaciones complementarias provenientes de fuentes expertas en violencia de género, siempre que proporcionen explicaciones rigurosas sobre las causas de esta clase de violencia. Igualmente, la incorporación de datos procedentes de los resultados de investigaciones científicas sobre la violencia machista y las aportaciones de fuentes no expertas se han tenido en cuenta si contribuyen a mejorar el conocimiento de la opinión pública sobre este problema social.

Asimismo, cabe mencionar que los elementos incluidos en este indicador no sólo son útiles para la medición de la labor de difusión del verdadero significado de la violencia machista, sino que también constituyen una propuesta dirigida a los medios de comunicación sobre los contenidos explicativos que, a nuestro juicio, deberían aparecer con cierta regularidad en la prensa escrita junto al relato objetivo de los hechos en los casos de agresiones machistas. Todo ello con el fin de contribuir a concienciar a la sociedad sobre este problema. 


\section{Análisis de los tres diarios}

\subsection{El País}

En las informaciones del diario El País son frecuentes las declaraciones oficiales de condena de los asesinatos machistas, pero no suelen ir acompañadas de comentarios adicionales que ayuden a comprender el verdadero origen de este problema, de manera que el rechazo institucional de la agresión se convierte en un elemento meramente anecdótico. Sólo en cuatro de las noticias seleccionadas de 2015, este diario incluye reflexiones que pueden mejorar el conocimiento de la ciudadanía sobre la violencia machista, lo que supone el $11,1 \%$ de las informaciones de la muestra. En la noticia del 2 de abril, además de aportar las cifras de mujeres asesinadas durante la última semana, se transmite la imagen de que esta clase de violencia ha alcanzado unas proporciones epidémicas, por medio de los datos de la Macroencuesta de Violencia contra la Mujer 2015: "una encuesta que señala que el 12,5\% de las mujeres mayores de 16 años que viven en España, unos 2,5 millones de mujeres, ha sufrido violencia física o sexual de sus parejas o exparejas a lo largo de su vida"3. La referencia explícita a los millones de mujeres agredidas en España produce un impacto mayor que la mera referencia al porcentaje de víctimas. Por otro lado, al especificar que la violencia machista incluye distintos tipos de agresiones, como la violencia física y la sexual, se transmite un concepto más amplio de este problema que el habitual en los mass media. De este modo, se produce cierta aproximación a la definición aceptada internacionalmente, que va más allá de la violencia física sufrida en el seno de las relaciones de pareja, e incluye también las agresiones sexuales y psicológicas producidas en el ámbito comunitario y la violencia contra las mujeres perpetrada o tolerada por el Estado.

En otra ocasión, en la noticia del 3 de junio, se dedica una parte importante del texto a reproducir las declaraciones de la directora del Instituto Andaluz de la Mujer, Carmen Cuello. Muchas de las declaraciones seleccionadas tienen que ver con el significado de la violencia machista y con la necesidad de que se implique toda la sociedad en la lucha contra este problema, como serían las siguientes:

- "Carmen Cuello, ha destacado 'la gravedad de un problema social que mata, que tiene su origen en el machismo y que precisa de una intervención global inmediata desde todos los ámbitos de la sociedad"”.

- "Cuello ha explicado que la violencia de género "es la manifestación más extrema' de la desigualdad hacia las mujeres".

- 'Ha animado a todas las personas a 'eliminar' en su vida 'toda actitud machista que contribuya a perpetuar, consentir o justificar' la desigualdad hacia las mujeres y la violencia de género en la que deriva dicha desigualdad"4.

\footnotetext{
3 Elpais.com, 02/04/2015.

4 Elpais.com, 03/06/2015.
} 
La selección de las declaraciones que aluden expresamente al significado de la violencia machista demuestra, por lo tanto, un importante grado de compromiso de las personas que firman la noticia con la causa de la igualdad de género. Asimismo, cabe destacar que esta información es ejemplar en cuanto al tratamiento de la violencia machista, puesto que no incluye comentarios sobre la situación personal del agresor o de la víctima que puedan inducir a pensar que estos elementos son la verdadera causa de la agresión. Tampoco incorpora declaraciones de vecinos o familiares que atribuyan la causa del asesinato a motivos misteriosos relacionados con el contexto del maltratador, o testimonios que elogien al agresor. Además, en la información se incluyen las declaraciones de condena de numerosos representantes institucionales, que transmiten una imagen de la violencia de género como un problema estructural que requiere la implicación de toda la sociedad y una mejor coordinación entre las distintas Administraciones.

Las declaraciones con valor pedagógico de Carmen Cuello vuelven a aparecer en la noticia del 27 de agosto, donde se afirma que la desigualdad de género y el machismo son la base de la violencia machista. Sin embargo, este mensaje resulta contradictorio con la presencia en la misma información de unas declaraciones desafortunadas del alcalde de Noalejo, quien se mostró "sorprendido y consternado", y definió al agresor y a la víctima como "unos vecinos "trabajadores, sin indicios de nada extraño" " 5 . Desde la perspectiva defendida en este trabajo, los comentarios que describen al agresor como una persona completamente normal, tranquila o pacífica, las alusiones a las buenas relaciones en el seno de la pareja que protagoniza la noticia, o las expresiones de sorpresa ante el desenlace de los acontecimientos, invitan a interpretar que la agresión ha sido producto de una causa externa excepcional que ha provocado un arrebato puntual o un ataque transitorio de locura del maltratador machista. Este hecho parece demostrar que los/as autores/as de las informaciones que incluyen testimonios de carácter pedagógico, no siempre poseen una formación adecuada en materia de igualdad de género o, al menos, no han prestado la suficiente atención a los principales manuales de recomendaciones sobre el tratamiento de la violencia machista en los medios. De este modo, la presencia de contenidos con valor didáctico podría deberse exclusivamente al azar o al fácil acceso a esta clase de declaraciones, y no a que la persona que ha elaborado la noticia esté comprometida con la causa de la igualdad de género.

En otra de las noticias de la muestra, la coordinadora provincial del Instituto Andaluz de la Mujer en Almería define esta clase de violencia como "la expresión máxima del machismo" y se refiere a ella como "problema social"6. Además, están presentes las declaraciones de la presidenta de la Asociación de Mujeres Juristas Themis, quien compara el "terrorismo machista" con el terrorismo etarra y el terrorismo yihadista. Consideramos que esta terminología también puede facilitar la comprensión del problema porque, como apunta Jay Sloan-Lynch, algunos/as filósofos/ as y autores/as feministas han cambiado la concepción popular del terrorismo y han sugerido que la violencia machista debería ser vista, con razón, como una forma de actividad terrorista: "Claudia Card, por ejemplo, sostiene que el maltrato doméstico es una forma de terrorismo frecuentemente ignorada, que crea y mantiene 'el domi-

\footnotetext{
5 Elpais.com, 27/08/2015.

6 Elpais.com, 31/12/2015.
} 
nio heterosexual masculino y la dependencia y servicio femeninos"' (Sloan-Lynch, 2012: 775). La violencia machista sería, por lo tanto, una forma de terrorismo dirigido a mantener el dominio y los privilegios masculinos a través del miedo.

\subsection{El Mundo}

En el caso de El Mundo, son cinco las noticias de la muestra que contribuyen a la divulgación de la noción de la violencia machista como una manifestación de la desigualdad de género. En la información del 8 de mayo se aprovechan las declaraciones de condena de los representantes de la Xunta de Galicia para señalar con claridad el origen de la violencia de género: "El Gobierno gallego [...] hace un llamamiento a toda la sociedad para que se rebele contra esta repudiable lacra y colabore para acabar con esta manifestación extrema de desigualdad que es la violencia de género"7.

En la noticia del 2 de abril, por otro lado, se aportan datos complementarios sobre el número de víctimas que recibieron protección en el País Vasco durante 2014: "En la comunidad autónoma vasca, un total de 3.325 mujeres víctimas de violencia machista reciben protección de la Ertzaintza a través de dispositivos electrónicos (teléfono Bortxa y pulsera), vigilancia o escolta personal"8. Esta información facilita la comprensión de la dimensión que ha alcanzado este problema en nuestro país. Además, la presencia explícita de la cifra de miles de mujeres afectadas en Euskadi traslada a los lectores y lectoras la idea de que la violencia machista es un fenómeno de enormes dimensiones, que incluye también a las mujeres heridas, acosadas o amenazadas. Por consiguiente, este problema no se limita a las decenas de casos de mujeres asesinadas anualmente, que es el único aspecto al que se presta atención desde los principales medios de comunicación del Estado español.

En otra ocasión, las palabras de la presidenta del Institut Català de les Dones permiten conocer cuál es la raíz de la violencia machista: "si la violencia machista todavía hace estragos en la sociedad es porque hay una base de desigualdades y discriminaciones entre hombres y mujeres que la alimentan y permiten', ha subrayado"9.

Finalmente, cabe destacar la información de El Mundo del 6 de octubre de 2015, por ser la que recoge un mayor número de declaraciones de fuentes expertas e institucionales, como las procedentes de Emakunde (Instituto Vasco de la Mujer) referidas a la necesidad de trabajar por la igualdad para prevenir la violencia de género, o las de la Asociación de Municipios Vascos (EUDEL) sobre la relación entre la desigualdad y la violencia machista o sobre los asesinatos como parte de un problema mucho más amplio.

Aunque en este diario las noticias con contenidos pedagógicos también suelen representar rigurosamente la violencia machista, en un número importante de informaciones se incluyen determinados datos sobre el agresor que pueden ser interpretados como un atenuante de la agresión, como son las referencias, a sus problemas mentales ("el hombre estaba recibiendo tratamiento psiquiátrico"10) o a su comportamiento conflictivo.

\footnotetext{
7 Elmundo.es, 08/05/2015.

8 Elmundo.es, 02/04/2015.

9 Elmundo.es, 12/08/2015.

${ }^{10}$ Elmundo.es, 05/12/2015.
} 


\section{3. $A B C$}

$A B C$ es el diario que incluye un menor número de reflexiones pedagógicas sobre la violencia machista durante 2015. Tal y como se puede apreciar en la tabla 1, sólo aparecen comentarios de este tipo en dos ocasiones, lo cual supone el 5,5\% de las noticias de la muestra. En una de estas informaciones se incluyen las declaraciones de la consejera de Igualdad, Salud y Políticas Sociales de Andalucía en funciones, María José Sánchez Rubio, en las que se expresa la estrecha relación entre desigualdad de género y violencia machista: "Según la consejera, esta muerte "solo se entiende desde ese criterio de desigualdad profunda que está detrás de la violencia machista', ante la que pide 'tolerancia cero' y aboga por "una intervención global inmediata" "11. En este caso, al igual que en la noticia de El País sobre este mismo asesinato, son numerosas las declaraciones institucionales de condena, lo cual invita a pensar que es la disponibilidad de dichas manifestaciones el principal motivo de su presencia en estos textos.

En la otra noticia mencionada, algunos de los testimonios coinciden con los presentes en la información publicada por El País el mismo día, como el de la coordinadora provincial del Instituto Andaluz de la Mujer en Almería. Junto a este último, aparecen mensajes enriquecedores, como el procedente de la Junta de Andalucía sobre la necesidad de modificar los estereotipos sexistas: "la Junta ha apostado por un refuerzo de la educación en igualdad como mejor 'herramienta de prevención', para transformar roles de género, fomentar nuevas masculinidades desvinculadas del dominio y el control y para promover relaciones sanas e igualitarias" 12 .

Finalmente, hemos de señalar que en las informaciones de los tres diarios analizados son frecuentes las declaraciones de condena y las cifras sobre mujeres asesinadas, pero no suelen ir acompañadas de explicaciones que ayuden a comprender el verdadero origen de este problema, de modo que la presencia de estos elementos, aunque es recomendada en algunos manuales sobre el tratamiento mediático de la violencia machista, carece de valor pedagógico de cara a los lectores y las lectoras.

Tabla 1. Proporción de noticias con valor pedagógico sobre la violencia machista.

\begin{tabular}{|l|c|c|c|c|}
\cline { 2 - 5 } \multicolumn{1}{c|}{} & ABC & El Mundo & El País & Total \\
\hline Porcentaje & $5,5 \%$ & $13,5 \%$ & $11,1 \%$ & $10,1 \%$ \\
\hline $\mathbf{N}^{\circ}$ noticias & 2 & 5 & 4 & 11 \\
\hline
\end{tabular}

Fuente: Elaboración propia.

\section{Conclusiones}

Durante el año 2015, las informaciones sobre violencia machista de los tres principales diarios de información general del Estado español apenas han incluido explicaciones que ayuden a mejorar el conocimiento de la ciudadanía sobre la violencia machista. Los comentarios de esta clase han estado presentes únicamente en el $11,1 \%$ de las noticias de El País y en el 13,5\% del diario El Mundo. En el caso de

\footnotetext{
${ }^{11}$ ABC.es, 03/06/2015.

${ }^{12}$ ABC.es, 31/12/2015.
} 
$A B C$, el porcentaje es todavía inferior, con un $5,5 \%$ de las noticias analizadas. Si la presencia de explicaciones de carácter pedagógico sobre la violencia de género en la prensa escrita es tan escasa, resulta muy complicado que quienes leen estas informaciones puedan vincular las agresiones que allí se describen con el orden patriarcal y la dominación masculina. Para que la opinión pública pueda conocer realmente por qué las mujeres son asesinadas, es necesario que los diarios transmitan mensajes explícitos sobre la relación directa entre la desigualdad de género y la violencia machista, en lugar de limitarse a seguir las recomendaciones de los manuales publicados sobre este tema y, como consecuencia, eliminar del relato de los hechos todos aquellos elementos que pueden ser interpretados erróneamente como la causa de la agresión (como podrían ser los problemas mentales o económicos del maltratador, las discusiones previas o el contexto de marginalidad de los protagonistas). Sin la presencia de estas explicaciones complementarias, es probable que persista la ignorancia de muchos de los lectores y lectoras de la información sobre el verdadero origen de este problema.

En este trabajo se comparte la opinión expresada en algunos manuales de recomendaciones sobre la representación mediática de la violencia machista, que defienden la conveniencia de incluir testimonios de fuentes expertas en la violencia de género en las noticias, declaraciones de condena, y también datos acumulativos que permitan reflejar la dimensión del fenómeno. Sin embargo, hemos podido comprobar que los tres diarios analizados han incluido las manifestaciones de condena o las cifras de víctimas de manera reiterada en sus informaciones, pero sin añadir comentarios explicativos sobre ellos, de modo que, habitualmente, los datos aportados se han convertido en un componente rutinario de carácter secundario. La presencia de estos elementos en solitario resulta escasamente útil para mejorar la comprensión de la violencia machista por parte de los lectores y las lectoras que carezcan de formación previa en materia de igualdad de género. En cuanto a las declaraciones procedentes de fuentes expertas, muchas de las que se reproducen en los medios de comunicación son intrascendentes, y no ayudan a difundir el significado real de esta clase de violencia. Para que la incorporación de estos elementos a la noticia resulte eficaz, las cifras de mujeres asesinadas deberían situarse en el contexto de un problema global que afecta a las mujeres de todo el mundo, y las declaraciones de condena tendrían que ir acompañadas de reflexiones sobre el origen de la violencia machista o sobre la necesidad de adoptar medidas preventivas para combatir el machismo. Igualmente, se deberían seleccionar preferentemente aquellas manifestaciones provenientes de fuentes expertas que amplíen el conocimiento ciudadano sobre este problema.

En general, en las noticias que incluyen comentarios con valor pedagógico, la violencia machista está representada adecuadamente. Sin embargo, se han detectado algunos hechos que podrían implicar que la presencia de dichos 
contenidos no se produce por una acción intencional de quienes elaboran las informaciones, sino que es casual, y que se debe a la mera disponibilidad de declaraciones de fuentes expertas, generadas en el contexto de determinadas agresiones machistas. Uno de los hechos señalados es la ausencia absoluta de explicaciones adicionales sobre la violencia machista elaboradas por el autor o autora de la información, y la escasez de datos científicos sobre este tema. Los contenidos pedagógicos se han limitado a la reproducción de las declaraciones procedentes de fuentes expertas disponibles durante las horas inmediatamente posteriores a la agresión que protagoniza la noticia concreta. Otro de los hechos detectados es el siguiente: aunque algunos/as periodistas llegan a firmar hasta cinco de las noticias seleccionadas, ninguno/a de ellos/ as incluye aportaciones con valor pedagógico en más de una información, y la mayoría de los y las periodistas no contribuyen a mejorar el conocimiento de la ciudadanía en ningún caso. Junto a esto último cabe destacar que un gran número de noticias siguen presentando elementos inapropiados que conllevan que la violencia machista sea tratada como una sucesión de casos aislados sin conexión entre sí y no como un problema social.

Todo lo recién expuesto nos lleva a concluir que no existe, en ninguno de los tres diarios analizados, un verdadero interés en la mejora del conocimiento que posee la ciudadanía sobre la violencia machista.

Desde la perspectiva que defiende que todos los géneros periodísticos interpretan la realidad, y que cualquier texto de este tipo lleva oculta una dosis de opinión, en el presente estudio se ha elaborado un indicador destinado a la medición del grado de contribución de los distintos formatos periodísticos a la divulgación de la noción de la violencia machista como una manifestación de la desigualdad de género. El análisis de esta función de la prensa se ha centrado, sin embargo, en un género informativo como la noticia con el objetivo de aprovechar la enorme audiencia potencial que poseen las informaciones. De esta manera, los contenidos de este indicador también constituyen una propuesta dirigida a quienes confeccionan las noticias sobre este tema, que incluye el tipo de explicaciones que deberían acompañar a los datos específicos referidos a cada caso particular o a las cifras desnudas de mujeres asesinadas. La presencia de los comentarios explicativos adicionales mencionados persigue el objetivo de situar estos casos concretos en el contexto de un problema global como es la violencia machista, y mejorar, de este modo, el conocimiento ciudadano sobre este asunto. 


\section{Referencias bibliográficas}

ARIZNABARRETA, Larraitz et al. (Equipo Investigación Género y Medios de Comunicación) (2006). Conclusiones. En: Tratamiento de la violencia de género en la prensa vasca. San Sebastián: Universidad de Deusto, pp. 177-181.

BANDRÉS, ELENA (2011). Propuesta para el tratamiento eficaz de la violencia de género. En: IC-Revista Científica de Información y Comunicación, $\mathrm{n}^{\circ} 8$, pp. 113-138.

BOSCH, Esperanza; FERRER, Victoria Aurora (2013). Nuevo modelo explicativo para la violencia contra las mujeres en la pareja: el modelo piramidal y el proceso de filtraje. En: Asparkía, nº 24, pp. 54-67.

BOSCH, Esperanza et al. (2013). La violencia contra las mujeres. El amor como coartada. Madrid: Anthropos.

BOSCH, Esperanza et al. (2012). Profundizando en el análisis del mito del amor romántico y sus relaciones con la violencia contra las mujeres en la pareja: análisis cualitativo. Año 2007-año 2011. Madrid: Ministerio de Sanidad, Servicios Sociales e Igualdad.

CARBALLIDO, Paula Carolina (2010). El proceso de construcción de la violencia contra las mujeres: medios de comunicación y movimiento feminista. Una aproximación desde la teoría del framing. Castellón de la Plana: Universitat Jaume I. Tesis doctoral.

COL·LEGI DE PERIODISTES DE CATALUNYA et al. (2004). Recomanacions sobre el tractament de la violència de gènere en els mitjans de comunicació. Barcelona: Institut Català de la Dona.

ESCRIBANO, María Isabel (2014). Encuadres de la violencia de género en la prensa escrita y digital, nacional y regional. La Verdad, La Opinión, El Mundo y El País desde la teoría del framing (2005-2010). Murcia: Universidad de Murcia. Tesis doctoral.

EUROPEAN COMMISSION (2010). Domestic Violence against Women Report. Special Eurobarometer 344 / Wave 73.2. Bruselas: Comisión Europea. Disponible en: http://ec.europa.eu/public_opinion/archives/ebs/ebs_344_en.pdf (consultado el 20 de julio de 2015).

FAIRCLOUGH, Norman (2003). El análisis crítico del discurso como método para la investigación en ciencias sociales. En: Ruth Wodak y Michael Meyer (comp.): Métodos de análisis crítico del discurso. Barcelona: Gedisa, pp. 179-204.

FERNÁNDEZ PARRATT, Sonia (2008). Géneros periodísticos en prensa. Quito: CIESPAL.

-- (2003). Introducción al reportaje: antecedentes, actualidad y perspectivas. Santiago de Compostela: Universidade de Santiago de Compostela.

MARIN, Flora; ARMENTIA, José Ignacio; CAMINOS, José (2011). El tratamiento informativo de las víctimas de violencia de género en Euskadi: Deia, El Correo, El País y Gara (2002-2009). En: Communication \& Society, vol. XXIV, $\mathrm{n}^{\mathrm{o}} 2$, pp. 435-466.

MARTÍNEZ ALBERTOS, José Luis (1992). Curso general de redacción periodística. Madrid: Paraninfo. 
MENÉNDEZ, Ma Isabel (2010). Representación mediática de la violencia de género. Análisis de la prensa balear (2004-2008). Palma: Universitat de les Illes Balears.

MILLET, Kate (1970). Sexual Politics. New York: Doubleday and Co.

ORGANIZACIÓN DE NACIONES UNIDAS (1995). Plataforma de Acción de Beijing. En: Informe de la Cuarta Conferencia Mundial sobre la Mujer. Pekín: ONU, pp. 6-143. Disponible en: http://www.un.org/womenwatch/daw/beijing/ pdf/Beijing\%20full\%20report\%20S.pdf (consultado el 22 de julio de 2015).

ROSELLÓ, Elena (2009). La violencia de género en los medios de comunicación. Alicante: Universidad de Alicante. Tesis doctoral.

SANZ, Begoña (2006). Entrevista y reportaje: dos modalidades eficaces en el tratamiento periodístico de la violencia de género. En: Larraitz Ariznabarreta et al. (Equipo Investigación Género y Medios de Comunicación): Tratamiento de la violencia de género en la prensa vasca. San Sebastián: Universidad de Deusto, pp. 97-113.

SCOLLON, Ron (2003). Acción y texto: para una comprensión conjunta del lugar del texto en la (inter)acción social, el análisis mediato del discurso y el problema de la acción social. En: Ruth Wodak y Michael Meyer (comp.): Métodos de análisis crítico del discurso. Barcelona: Gedisa, pp. 205-266.

SLOAN-LYNCH, Jay (2012). Domestic Abuse as Terrorism. En: Hypatia, vol. 27, $\mathrm{n}^{\circ}$ 4,pp. 774-790.

VAN DIJK, Teun A. (2003). La multidisciplinariedad del análisis crítico del discurso: un alegato en favor de la diversidad. En: Ruth Wodak y Michael Meyer (comp.): Métodos de análisis crítico del discurso. Barcelona: Gedisa, pp. 143-178. 